\title{
Criminologie
}

\section{Culpabilité chez les enfants victimes d'agression sexuelle} Le rôle médiateur des stratégies d'évitement sur l'anxiété et l'estime de soi

\section{Sense of guilt in sexually abused children}

The mediating role of avoidance coping on anxiety and self-esteem

\section{Culpabilidad en los niños víctimas de agresión sexual El rol mediador de las estrategias de evitación sobre la ansiedad y la autoestima}

\author{
Amélie Gauthier-Duchesne, Martine Hébert et Marie-Ève Daspe
}

Volume 50, numéro 1, printemps 2017

L'agression sexuelle commise sur des mineurs : les victimes, les auteurs

URI : https://id.erudit.org/iderudit/1039801ar

DOI : https://doi.org/10.7202/1039801ar

Aller au sommaire du numéro

Éditeur(s)

Les Presses de l’Université de Montréal

ISSN

0316-0041 (imprimé)

1492-1367 (numérique)

Découvrir la revue

Citer cet article

Gauthier-Duchesne, A., Hébert, M. \& Daspe, M.-È. (2017). Culpabilité chez les enfants victimes d'agression sexuelle : le rôle médiateur des stratégies d'évitement sur l'anxiété et l'estime de soi. Criminologie, 50(1), 181-201. https://doi.org/10.7202/1039801ar

\section{Résumé de l'article}

Des études antérieures relèvent que le sentiment de culpabilité est un facteur associé aux répercussions de l'agression sexuelle (AS) chez les survivants adultes (Cantón-Cortés, Cantón, Justicia et Cortés, 2011). Toutefois, très peu d'études ont exploré le rôle potentiel du sentiment de culpabilité sur les symptômes chez les enfants victimes. L'objectif de cette recherche est d'étudier le rôle médiateur de l'évitement dans la relation entre le sentiment de culpabilité et les symptômes associés à l'AS (anxiété et estime de soi). L'échantillon est composé de 447 enfants victimes d'AS (319 filles et 128 garçons), âgés de 6 à 12 ans. Les résultats des analyses acheminatoires indiquent que les enfants révélant davantage de culpabilité par rapport à la situation d'AS présentent un niveau plus élevé d'anxiété et une plus faible estime d'eux-mêmes. Un effet indirect a également été observé et montre que le sentiment de culpabilité est lié à l'utilisation de stratégies d'évitement, qui en retour exacerbent les symptômes d'anxiété et contribuent à une plus faible estime de soi. Le modèle, qui s'ajuste aux données de manière équivalente pour les filles et les garçons, permet d'expliquer $24,4 \%$ de la variance des symptômes d'anxiété et $11,2 \%$ de la variance de l'estime de soi. Ces résultats laissent entendre que le sentiment de culpabilité pourrait constituer une cible d'intervention pertinente pour les enfants victimes d'AS. 


\title{
Culpabilité chez les enfants victimes d'agression sexuelle
}

\author{
Le rôle médiateur des stratégies d'évitement \\ sur l'anxiété et l'estime de soi ${ }^{1}$
}

\author{
Amélie Gauthier-Duchesne, M.A. ${ }^{2}$ \\ Candidate au doctorat en sexologie \\ Université du Québec à Montréal \\ gauthier-duchesne.amelie@courrier.uqam.ca \\ Martine Hébert, Ph. D. \\ Professeure au département de sexologie \\ Université du Québec à Montréal \\ hebert.m@uqam.ca \\ Marie-Ève Daspe, Ph. D. \\ Stagiaire postdoctorale \\ Université du Québec à Montréal \\ daspe.marie-eve@courrier.uqam.ca
}

RÉSUMÉ - Des études antérieures relèvent que le sentiment de culpabilité est un facteur associé aux répercussions de l'agression sexuelle (AS) chez les survivants adultes (Cantón-Cortés, Cantón, Justicia et Cortés, 2011). Toutefois, très peu d'études ont exploré le rôle potentiel du sentiment de culpabilité sur les symptômes chez les enfants victimes. L'objectif de cette recherche est d'étudier le rôle médiateur de l'évitement dans la relation entre le sentiment de culpabilité et les symptômes associés à l'AS (anxiété et estime de soi). L'échantillon est composé de 447 enfants victimes d'AS (319 filles et 128 garçons), âgés de 6 à 12 ans. Les résultats des

1. Cette recherche a été réalisée grâce à une subvention des Instituts de recherche en santé du Canada $\left(\mathrm{n}^{\circ} 77614\right)$ octroyée à Martine Hébert. La première auteure tient à remercier les organismes lui ayant octroyé des bourses d'études supérieures, soit le Conseil de recherches en sciences humaines du Canada, les Fonds de recherche du Québec - Société et culture ainsi que la Chaire interuniversitaire Marie-Vincent sur les agressions sexuelles envers les enfants. Nous tenons également à remercier les familles ayant participé à ce projet et les professionnels des centres d'intervention impliqués.

2. Département de sexologie, Université du Québec à Montréal, C. P. 8888, succ. Centre-ville, Montréal (Québec), Canada, H3C 3P8. 
analyses acheminatoires indiquent que les enfants révélant davantage de culpabilité par rapport à la situation d'AS présentent un niveau plus élevé d'anxiété et une plus faible estime d'eux-mêmes. Un effet indirect a également été observé et montre que le sentiment de culpabilité est lié à l'utilisation de stratégies d'évitement, qui en retour exacerbent les symptômes d'anxiété et contribuent à une plus faible estime de soi. Le modèle, qui s'ajuste aux données de manière équivalente pour les filles et les garçons, permet d'expliquer $24,4 \%$ de la variance des symptômes d'anxiété et $11,2 \%$ de la variance de l'estime de soi. Ces résultats laissent entendre que le sentiment de culpabilité pourrait constituer une cible d'intervention pertinente pour les enfants victimes d'AS.

MOTS CLÉS • Enfants victimes d'agression sexuelle, sentiment de culpabilité, stratégies d'évitement, anxiété, estime de soi.

\section{Introduction}

L'agression sexuelle (AS) représente un problème social grave qui touche principalement les femmes et les enfants. Les méta-analyses réalisées à partir d'échantillons de différents pays révèlent qu'une fille sur cinq et un garçon sur dix ont été victimes d'AS avant l'âge de 18 ans (Stoltenborgh, van Ijzendoorn, Euser et Bakermans-Kranenburg, 2011). Au Québec, on retrouve des taux de prévalence équivalents concernant les AS vécues durant l'enfance (Tourigny et Baril, 2011).

Vu l'ampleur du phénomène, on observe une augmentation de l'intérêt scientifique depuis les trente dernières années en ce qui a trait aux AS vécues durant l'enfance. Plusieurs recherches ont donc permis de déterminer des conséquences négatives associées à une histoire de victimisation sexuelle durant l'enfance. Ainsi, l'AS est maintenant clairement reconnue comme un facteur de risque important pour les tentatives de suicide, l'abus d'alcool et de drogues et la revictimisation (Maniglio, 2009). Bien que moins documentés que ces conséquences à long terme, de nombreux symptômes ont également été observés chez les enfants victimes d'AS. Les enfants ayant dévoilé une situation d'AS sont susceptibles de présenter des symptômes de stress posttraumatique (SSPT; Hébert, Langevin et Daigneault, 2016), ainsi que des symptômes dépressifs et anxieux (Hébert, 2011). Certains enfants peuvent également exprimer davantage de problèmes de comportement extériorisés, comme de la colère, de l'agressivité ainsi que des comportements sexualisés problématiques (Gagnon et Tourigny, 2011 ; Hébert, 2011). Les enfants victimes d'AS sont également plus à risque de développer une faible estime d'eux-mêmes (Heflin et Deblinger, 2007). 


\section{Sentiment de culpabilité}

La multiplicité et la variabilité des symptômes soulignent l'importance de mieux comprendre les trajectoires des enfants victimes d'AS. De surcroit, il semble primordial d'étudier les facteurs qui contribuent à l'apparition de ces symptômes afin de mieux adapter les interventions offertes auprès de cette population, et ainsi diminuer les conséquences vécues par les victimes d'AS durant l'enfance. Parmi ces facteurs, quelques études se sont penchées sur le sentiment de culpabilité. Lorsqu'une personne fait face à une expérience de vie défavorable, comme une AS, elle peut tenter d'attribuer des causes qui expliqueraient pourquoi elle se trouve dans cette situation (Feiring et Cleland, 2007). Ces attributions sont généralement décrites comme étant internes (p. ex., s'attribuer le blâme) ou externes (p. ex., blâmer l'agresseur). Chez les victimes d'AS, les attributions internes de blâme peuvent être exacerbées par les commentaires et les comportements de l'agresseur et de l'entourage de la victime lors du dévoilement pouvant blesser ou stigmatiser l'enfant (Finkelhor et Browne, 1985). Un sentiment de culpabilité élevé peut donc découler de cette attribution interne de blâme. Chez la population générale, il est reconnu que les attributions internes à la suite d'événements de vie adverses sont associées à davantage de détresse psychologique, notamment des symptômes anxieux (Martin et Dahlen, 2005) et une faible estime de soi (Major, Kaiser et McCoy, 2003).

Quelques études empiriques ont étudié le sentiment de culpabilité à titre de facteur prédicteur ou médiateur des conséquences de l'AS. Par exemple, Daigneault, Tourigny et Hébert (2006) ont observé que les attributions internes de blâme propres à l'AS contribuent à prédire différents symptômes chez des adolescentes victimes, dont des symptômes anxieux et dépressifs. Une étude de Feiring et Cleland (2007) a également révélé que les attributions internes, comparativement aux attributions externes, sont un prédicteur des symptômes dépressifs chez de jeunes victimes âgées de 8 à 15 ans. Cependant, ces recherches ont été conduites principalement auprès d'adolescents et tiennent compte uniquement des attributions causales et non du sentiment qui en découle, ce qui empêche de dresser un portrait juste du sentiment de culpabilité vécu par les enfants d'âge scolaire victimes d'AS. Il importe également de faire la distinction entre le sentiment de culpabilité et le sentiment de honte. Ce dernier renvoie davantage à une évaluation négative de soi, se traduisant par exemple par l'impression de se sentir petit, inutile, impuissant et exposé de manière négative aux autres 
(Tangney, Stuewig et Mashek, 2007). Le sentiment de culpabilité réfère plutôt à une évaluation émotionnelle négative de ses comportements et de ses actions, et non de sa personne (Lewis, 1971). La honte peut être observée chez les victimes d'AS lorsqu'elles tentent de se cacher en parlant de leur expérience traumatique (Feiring et Taska, 2005). Les enfants peuvent se sentir honteux d'avoir été impliqués dans des comportements sexuels jugés inappropriés (Feiring, Taska et Chen, 2002), alors que la culpabilité est associée au regret d'avoir fait (ou de ne pas avoir fait) certains gestes.

Le sentiment de culpabilité vécu chez les victimes peut aussi être influencé par les caractéristiques de l'AS. Plusieurs auteurs ont observé que les situations d'AS impliquant des gestes plus sévères, c'est-à-dire des gestes de pénétration, contribuent à des niveaux plus élevés de sentiments de culpabilité (Cantón-Cortés, Cantón, Justicia et Cortés, 2011; Feiring et Cleland, 2007; Quas, Goodman et Jones, 2003). Cependant, Ullman, Townsend, Filipas et Starzynski (2007) soulèvent des résultats différents en observant que le degré de sévérité de l'AS est négativement associé au sentiment de culpabilité. Cette dernière recherche a toutefois été conduite auprès de femmes ayant été victimes durant l'adolescence ou à l'âge adulte. Les femmes adultes victimes d'AS impliquant une pénétration pourraient ressentir moins de culpabilité, car les gestes qu'elles ont subis seraient conformes aux croyances sociales qui définissent le viol (Ullman et al., 2007). Les victimes d'AS très sévères durant l'enfance seraient quant à elles plus susceptibles de se percevoir comme responsables de la situation (Feiring et Cleland, 2007). En effet, des gestes plus sévères pourraient amener les enfants à croire qu'il y a quelque chose chez eux qui conduit à l'AS (Feiring et Cleland, 2007). Des contradictions émergent également quant aux résultats explorant le lien avec l'agresseur: certains relatent que le fait d'avoir une relation de proximité avec l'agresseur est un facteur prédictif du sentiment de culpabilité (Quas et al., 2003), alors que d'autres n'observent aucun lien significatif (Cantón-Cortés et al., 2011). Cette disparité pourrait être expliquée par la complexité de l'attribution du blâme lorsque l'agresseur est une personne aimée par l'enfant (Feiring et Cleland, 2007). Enfin, il est possible de penser que la fréquence de l'AS pourrait contribuer au sentiment de culpabilité chez les victimes (Cantón-Cortés et al., 2011; Quas et al., 2003), car celles-ci pourraient se sentir coupables de ne pas avoir été en mesure de mettre un terme à la situation abusive. Ces contradictions présentes dans la littérature 
scientifique soulignent la pertinence d'étudier le lien entre les caractéristiques de l'AS et le sentiment de culpabilité chez les victimes.

\section{Stratégies d'évitement}

Un autre facteur pouvant moduler l'intensité des symptômes vécus par les enfants victimes d'AS réfère aux stratégies d'adaptation. Celles-ci permettent aux individus de résoudre un problème ou une difficulté à la suite d'un événement de vie adverse. Les stratégies d'adaptation sont généralement décrites selon deux catégories, soit les stratégies d'approche et les stratégies d'évitement (Lazarus, 1993). La première catégorie réfère aux actions qui permettent à l'individu d'affronter l'événement stressant (p. ex., parler de ses sentiments, résolution de problèmes); elles sont généralement associées à un meilleur ajustement à long terme. Les stratégies d'évitement réfèrent quant à elles aux comportements permettant à une personne de ne pas être exposée à des informations pouvant être difficiles à gérer (p. ex., éviter de penser à ce qui est arrivé, distanciation). L'utilisation de ce type de stratégies contribue à l'apparition de problèmes de comportement intériorisés (retrait, anxiété, dépression) chez les jeunes victimes (Hébert, Tremblay, Parent, Daignault et Piché, 2006; Hébert, 2014). Cantón-Cortés et ses collègues (2011) ont de leur côté observé l'effet médiateur des stratégies d'évitement entre le sentiment de culpabilité et les SSPT chez de jeunes femmes âgées de 18 à 24 ans ayant été victimes d'AS avant l'âge de 14 ans. Puisque l'attribution interne de blâme peut être difficile à gérer, les victimes pourraient avoir recours à des stratégies d'évitement afin d'échapper aux pensées et aux sentiments douloureux. D'autres recherches sont nécessaires afin d'étudier l'effet médiateur des stratégies d'évitement entre le sentiment de culpabilité et d'autres symptômes associés aux AS vécues durant l'enfance.

\section{Différences liées au genre}

L'analyse des études publiées dans le domaine indique que malgré qu'un garçon sur dix serait victime d'AS durant l'enfance (Stoltenborgh et al., 2011 ; Tourigny et Baril, 2011), les garçons et les hommes sont, dans la vaste majorité des études, sous-représentés dans les échantillons, voire absents (Maikovich-Fong et Jaffee, 2010; Villeneuve, Cyr et Hébert, 2011). Parmi les études qui se sont penchées sur les différences entre 
les garçons et les filles victimes d'AS, on y décèle des résultats divergents (Villeneuve et al., 2011). Par exemple, en ce qui concerne le sentiment de culpabilité, Ullman et Filipas (2005) recensent que les femmes victimes d'AS durant l'enfance se sentiraient davantage coupables que les hommes victimes, alors que Feiring et Cleland (2007) n'ont observé aucune différence significative entre les garçons et filles, justifiant ainsi la nécessité d'explorer les différences de genre dans les études portant sur les AS vécues durant l'enfance.

L'objectif de cette recherche est d'étudier le rôle médiateur de l'évitement dans la relation entre le sentiment de culpabilité et les symptômes associés à l'AS (anxiété et estime de soi) chez les enfants victimes. Au sein du modèle étudié, les caractéristiques de l'AS (sévérité des gestes faits, fréquence et identité de l'agresseur) sont considérées comme étant des prédicteurs du sentiment de culpabilité vécu par la victime. Il est attendu que des gestes plus sévères, une fréquence élevée et une relation de proximité avec l'agresseur soient des facteurs associés à un plus grand sentiment de culpabilité. Ce sentiment de culpabilité devrait être positivement associé aux symptômes anxieux et négativement associé à l'estime de soi, via une plus grande utilisation de stratégies d'évitement. La présence de différences liées au genre au sein de ce modèle sera également examinée de façon exploratoire. Au regard des études précédemment recensées, il semble pertinent d'étudier le sentiment de culpabilité propre à l'AS vécu chez les enfants victimes d'AS, en tenant compte du genre de l'enfant. Mieux comprendre les symptômes présents chez cette population permettra d'adapter les interventions offertes et ainsi diminuer les conséquences chez les jeunes victimes.

\section{Méthodologie}

\section{Participants}

L'échantillon comprend 447 enfants (319 filles et 128 garçons), âgés de 6 à 12 ans $(M=8,99 ; E . T .=2,05)$, qui ont été victimes d'AS. Les participants ont été recrutés lors de l'évaluation initiale dans différents sites offrant des services spécialisés, soit la Clinique pédiatrique sociojuridique du Centre hospitalier universitaire (CHU) Sainte-Justine, le Centre d'expertise en agression sexuelle Marie-Vincent, le Centre d'intervention en abus sexuels pour la famille et Parents-Unis Repentigny Lanaudière. Le Tableau 1 présente les caractéristiques sociodémographiques des enfants et de leur famille. 
TA BLEAU 1

Caractéristiques sociodémographiques des participants

\begin{tabular}{|l|c|}
\hline Caractéristiques & $\begin{array}{c}\text { Participants } \\
(n=447)\end{array}$ \\
\hline Genre de l'enfant & \\
\hline Fille & $71,4 \%$ \\
\hline Garçon & $28,6 \%$ \\
\hline Structure familiale & \\
\hline Famille d'origine & $17,8 \%$ \\
\hline Famille monoparentale & $42,5 \%$ \\
\hline Famille reconstituée & $27,3 \%$ \\
\hline Famille d'accueil ou en Centre jeunesse & $12,4 \%$ \\
\hline Revenu familial & \\
\hline Moins de 20 000 \$ & $32,9 \%$ \\
\hline 20000 à 39 999 \$ & $27,7 \%$ \\
\hline 40 000 à 59 999 \$ & $16,8 \%$ \\
\hline 60 000 \$ et plus & $22,6 \%$ \\
\hline Degré de scolarité de la mère & \\
\hline Études primaires & $4,8 \%$ \\
\hline Études secondaires & $45,1 \%$ \\
\hline Études collégiales & $38,0 \%$ \\
\hline Études universitaires & $12,1 \%$ \\
\hline Degré de scolarité du père & \\
\hline Études primaires & $9,9 \%$ \\
\hline Études secondaires & $26,9 \%$ \\
\hline Études collégiales & $13,8 \%$ \\
\hline Études universitaires & \\
\hline Appartenance ethnoculturelle & \\
\hline Canadienne ou québécoise & \\
\hline Autre & \\
\hline
\end{tabular}

\section{Mesures}

\section{Caractéristiques de l'AS}

Une version adaptée du History of Victimization Form (HVF; Parent et Hébert, 2006) a été utilisée afin de collecter les informations quant aux caractéristiques de l'AS à partir des renseignements disponibles dans le 
dossier médical ou clinique de l'enfant. La sévérité des gestes posés par l'agresseur a été cotée à l'aide d'une échelle de 3 points: $1=$ exhibitionnisme (exposition des parties génitales de l'agresseur sans toucher), voyeurisme (exposition des parties génitales de l'enfant sans toucher), baisers, exposition à du matériel pornographique, contact physique par-dessus les vêtements; 2 = contact physique sous les vêtements, attouchements génitaux; et $3=$ contact oral-génital, pénétration ou tentative de pénétration (digitale, orale, vaginale, anale ou avec un objet). La fréquence de l'AS a été catégorisée comme étant $1=$ épisode unique; 2 = plusieurs événements; et 3 = répétitive ou chronique (plus de 6 mois). Une échelle de 4 points a été utilisée pour coter l'identité de l'agresseur : 1 = un membre de la famille immédiate (parent, fratrie, conjoint du parent, enfant du conjoint du parent); 2 = un membre de la famille éloignée (grand-parent, oncle, tante, cousin); $3=$ une connaissance de l'enfant (p. ex., gardien, voisin, ami de la famille); et $4=$ un inconnu.

\section{Sentiment de culpabilité}

Les enfants ont rempli le Children's Impact of Traumatic Events Scale II (CITES-II; Wolfe, 2002) qui comprend 3 items sur le sentiment de culpabilité propre à la situation d'AS. Ces items sont: «Je pense que ce qui est arrivé est de ma faute», "J'ai l'impression d'avoir causé des problèmes à plusieurs personnes» et "Je me sens coupable de ce qui est arrivé». Chaque item inclut les choix de réponse suivants: $0=$ Faux, $1=$ Un peu vrai et $2=$ Très vrai. Le score total du sentiment de culpabilité varie de 0 à 6 et le coefficient de consistance interne obtenu lors de la présente étude est satisfaisant $(\alpha=0,71)$.

\section{Stratégies d'évitement}

Une version traduite et abrégée du Self-Report Coping Scale (SRCS; Causey et Bubow, 1992; Hébert, Parent et Daignault, 2007) a été passée aux enfants. L'échelle contient 20 items concernant des stratégies d'approche et d'évitement liées à la situation d'AS. Pour chaque stratégie, les choix de réponse sont $1=$ Jamais, $2=$ Presque jamais, $3=$ Quelquefois, $4=$ La plupart du temps et $5=$ Toujours. Le score total de la sous-échelle des stratégies d'évitement varie de 4 à 20 . "Je fais comme si rien n'était arrivé» et "Je me dis que ce qui est arrivé n'est pas important» sont des exemples d'items liés aux stratégies d'évitement. La consistance interne de la version française de cette sous-échelle 
est adéquate $(\alpha>0,72$; Hébert et al., 2007). Dans le cadre de cette recherche, le coefficient alpha est de 0,71.

\section{Symptômes anxieux}

Les 11 items de la sous-échelle d'inquiétude-hypersensibilité du Revised Children's Manifest Anxiety Scale (RCMAS; Reynolds et Richmond, 1978) ont été remplis par les enfants. Cette échelle a été traduite par Turgeon et Chartrand (2003). Les choix de réponse sont $0=$ Faux et $1=$ Vrai, et le score total varie de 0 à 11 . Des exemples d'items sont: «Je deviens nerveux/nerveuse quand les choses ne vont pas comme je veux» et «Je m'inquiète de ce que mes parents peuvent me dire». La consistance interne obtenue par Turgeon et Chartrand (2003) est adéquate et égale à celle obtenue dans le cadre de la présente étude $(\alpha=0,81)$.

\section{Estime de soi}

L'échelle de l'estime de soi globale du Self-Perception Profile for Children (SPPC; Harter, 1985) a été remplie par les enfants. Cette échelle a été traduite par Hébert et Parent (1995). Pour chacun des 6 items, les enfants devaient choisir, dans un premier temps, lequel des deux types d'enfants lui correspond le plus (p. ex: "Certains enfants aiment le genre de personne qu'ils sont MAIS d'autres enfants aimeraient être quelqu'un d'autre») et, dans un deuxième temps, si ce type d'enfants est un peu ou tout à fait comme lui. Un score de 1 à 4 est attribué pour chaque item ( 1 = faible estime de soi et $4=$ estime de soi élevée). Le score total de cette échelle est de 6 à 24 . La consistance interne obtenue par Harter (1985) est adéquate $(\alpha>0,78)$. Dans la présente étude, la consistance interne est $\alpha=0,68$.

\section{Procédures}

Les enfants ont été sollicités pour participer à la recherche lors de leur visite au centre spécialisé. Pour ceux qui ont accepté de participer, le formulaire de consentement a été expliqué et signé par le parent. La confidentialité a été assurée aux participants. L'enfant a rempli le questionnaire seul avec une assistante de recherche. La passation du questionnaire durait environ une heure. Ce projet de recherche a été approuvé par le comité d'éthique de la recherche du CHU Sainte-Justine et du comité institutionnel d'éthique de la recherche de l'Université du Québec à Montréal. 


\section{Stratégies d'analyses}

Dans un premier temps, à l'aide du logiciel SPSS version 20.0, des analyses descriptives ont été réalisées afin de documenter les caractéristiques des situations d'AS vécues par les enfants. Dans un deuxième temps, des analyses de corrélation ont été effectuées afin de présenter les relations entre les différentes variables à l'étude. Dans un troisième temps, des analyses acheminatoires, réalisées avec le logiciel Mplus version 7.0 (Muthén et Muthén, 1998-2015), ont été effectuées afin de tester le modèle de médiation. Différents indices ont été utilisés afin de vérifier si le modèle spécifié représente les données de manière adéquate. Un chi carré non significatif ou un ratio du chi carré sur les degrés de liberté inférieur à 3 (Jöreskog et Sörbom, 1993), un CFI (Comparative Fit Index) supérieur à 0,95 (Hu et Bentler, 1999) et un RMSEA (Root Mean Square Error of Approximation) plus petit que 0,06, avec un intervalle de confiance (IC) à $90 \%$ se situant entre 0,00 et 0,08 (Hu et Bentler, 1999), indiquent tous un bon ajustement.

Afin d'examiner la contribution des stratégies d'évitement dans l'association entre le sentiment de culpabilité et les symptômes d'anxiété et d'estime de soi, des effets indirects sont estimés. Pour ce faire, le produit des coefficients de régression de l'évitement sur la culpabilité et de l'anxiété sur l'évitement est calculé. Le même processus est effectué pour le lien indirect entre la culpabilité, l'évitement et l'estime de soi. Afin de vérifier si ces effets indirects sont significatifs, des IC bootstrap à $95 \%$ sont estimés (MacKinnon et Fairchild, 2009). Cette méthode est basée sur une distribution des produits de coefficients et génère des IC pour la valeur réelle des coefficients des effets indirects. Enfin, des analyses multigroupes sont réalisées afin de vérifier l'invariance du modèle spécifié selon le genre des enfants victimes.

\section{Résultats}

Les analyses descriptives (voir Tableau 2) indiquent que la majorité des enfants composant l'échantillon ont vécu des situations d'AS impliquant des gestes très sévères (pénétration ou tentative de pénétration). Plus de la moitié des enfants auraient été abusés par un membre de leur famille immédiate.

Les corrélations présentées dans le Tableau 3 indiquent que le sentiment de culpabilité et les stratégies d'évitement sont associés à davantage 
de symptômes anxieux et à une faible estime de soi. La sévérité de l'AS est associée à un plus grand sentiment de culpabilité vécu par l'enfant, mais les autres caractéristiques de l'AS, soit la fréquence et l'identité de l'agresseur, ne sont pas corrélées au sentiment de culpabilité. Ces caractéristiques ne seront donc pas incluses dans le modèle.

TA BLEA U 2

Caractéristiques des situations d'AS chez les enfants victimes

\begin{tabular}{|l|c|}
\hline Caractéristiques & Participants $(n=447)$ \\
\hline Sévérité de l'AS & \\
\hline AS moins sévère & $10,7 \%$ \\
\hline AS sévère & $27,7 \%$ \\
\hline AS très sévère & $61,6 \%$ \\
\hline Fréquence de l'AS & \\
\hline Épisode unique & $23,5 \%$ \\
\hline Quelques événements & $40,6 \%$ \\
\hline Répétitive ou chronique & $35,9 \%$ \\
\hline Identité de l'agresseur & \\
\hline Famille immédiate & $52,6 \%$ \\
\hline Famille élargie & $20,0 \%$ \\
\hline Connaissances & $25,4 \%$ \\
\hline Inconnu & $2,0 \%$ \\
\hline
\end{tabular}

Modèle de médiation

Des analyses acheminatoires ont été réalisées afin d'étudier le rôle médiateur de l'évitement dans la relation entre le sentiment de culpabilité et les symptômes associés à l'AS (anxiété et estime de soi). Puisque certaines variables n'étaient pas distribuées de manière normale, une méthode d'estimation robuste a été utilisée. Cette méthode permet de gérer les données manquantes selon la méthode d'estimation par maximum de vraisemblance (Muthén et Muthén, 1998-2015) et d'obtenir des indices d'ajustement adaptés aux données anormalement distribuées (Yuan et Bentler, 2000). Le modèle de médiation (voir Figure 1) s'adapte adéquatement aux données recueillies $\left(\chi^{2}(3)=3,295, p=0,35\right.$; $\chi^{2} / d f=1,10 ;$ CFI $=1,00 ;$ RMSEA $=0,02$ avec un IC $90 \%=[0,00$ à 0,08$])$. 
TABLEAU 3

Corrélations, moyennes et écarts types des variables à l'étude

\begin{tabular}{|c|c|c|c|c|c|c|c|c|}
\hline Variables & 1 & 2 & 3 & 4 & 5 & 6 & Moyennes & Écarts types \\
\hline 1. Culpabilité & & & & & & & 1,50 & 1,77 \\
\hline 2. Évitement &, $302^{* * *}$ & & & & & & 10,20 & 3,03 \\
\hline 3. Anxiété & $295^{* * *}$ &, $470 * * *$ & & & & & 5,47 & 3,21 \\
\hline 4. Estime de soi &,$- 256^{* * *}$ &,$- 288^{* * *}$ &,$- 367 * * *$ & & & & 18,62 & 3,97 \\
\hline 5. Sévérité de l'AS & , 103* & 092 & 017 &,- 068 & & & 2,51 & 0,68 \\
\hline 6. Fréquence de l'AS & ,005 & 029 &,- 031 &,- 072 &, $220^{* * *}$ & & 2,12 & 0,76 \\
\hline 7. Identité de l'agresseur &,- 067 &, 001 &, 005 & ,004 &,- 077 &,$- 191^{* * *}$ & 1,77 & 0,90 \\
\hline
\end{tabular}

Notes: ${ }^{*} p<0,05 ;{ }^{* *} p<0,01 ;{ }^{* \star \star} p<0,001$. 
FIG URE 1

Modèle prédictif de l'anxiété et de l'estime de soi chez les enfants victimes d'AS

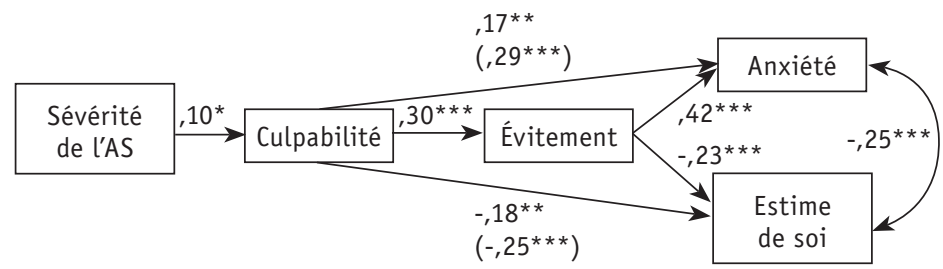

Notes: Les coefficients du modèle sont standardisés. Les coefficients de régression inscrits entre parenthèses sont ceux obtenus avant d'intégrer la variable évitement comme médiateur au modèle. Le modèle explique $24,4 \%$ de la variance des symptômes d'anxiété et $11,2 \%$ de la variance de l'estime de soi.

${ }^{*} p<0,05 ;{ }^{* *} p<0,01 ;{ }^{* *} p<0,001$.

Les résultats indiquent que la sévérité de l'AS est associée au sentiment de culpabilité vécu par l'enfant. De plus, le sentiment de culpabilité vécu par l'enfant prédit les symptômes anxieux de manière directe et indirecte via les stratégies d'évitement $(b=0,23$ avec un IC $95 \%=$ $[0,15$ à 0,33$])$. La proportion de l'effet indirect du sentiment de culpabilité sur l'anxiété via les stratégies d'évitement est de $43 \%$. Finalement, le sentiment de culpabilité prédit également l'estime de soi, avec un effet médiateur des stratégies d'évitement $(b=-0,16$ avec un IC $95 \%$ $=[-0,25$ à $-0,09])$. La proportion de l'effet indirect du sentiment de culpabilité sur l'estime de soi par les stratégies d'évitement est de $27 \%$. Le modèle explique $24,4 \%$ de la variance des symptômes d'anxiété et $11,2 \%$ de la variance de l'estime de soi chez les enfants victimes d'AS.

\section{Différences liées au genre}

Dans le but d'explorer la présence de différences entre les garçons et les filles, des analyses multigroupes sont réalisées afin d'examiner l'invariance du modèle selon le genre de la victime. Pour ce faire, un modèle multigroupe dans lequel l'ensemble des paramètres (liens structuraux, variances et covariances) des garçons et des filles sont contraints à égalité est comparé à un modèle dans lequel ces paramètres sont estimés librement. La différence entre le chi carré du modèle sans contrainte et le chi carré du modèle présentant des contraintes d'égalité n'est pas significative $\left(\Delta \chi^{2}(6)=7,19, p=0,30\right)$, ce qui indique que le modèle spécifié s'ajuste de manière équivalente aux données des filles et des garçons. 


\section{Discussion}

La présente étude avait pour but d'étudier le rôle médiateur des stratégies d'évitement dans la relation entre le sentiment de culpabilité et les symptômes associés à l'AS (anxiété et estime de soi) chez les enfants victimes d'AS. Les résultats ont démontré que le sentiment de culpabilité est associé à un plus haut niveau d'anxiété et à une plus faible estime de soi. Ce lien est également observé chez la population générale adulte (Major et al., 2003; Martin et Dahlen, 2005). Des recherches antérieures conduites auprès d'adolescents victimes d'AS avaient montré que le sentiment de culpabilité était un prédicteur de différentes difficultés associées au trauma, dont des symptômes de stress post-traumatique, dépressifs et anxieux (Cantón-Cortés et al., 2011; Daigneault et al., 2006; Feiring et Cleland, 2007). En plus des effets directs observés, les analyses ont permis de constater que l'évitement joue un rôle médiateur entre le sentiment de culpabilité et les symptômes associés à l'AS. Ces résultats sont cohérents avec ceux recensés chez les adultes victimes d'AS durant l'enfance (Cantón-Cortés et al., 2011). Il est d'ailleurs reconnu que l'utilisation de stratégies d'évitement est liée à la détresse, et ce, tant pour la population clinique que spécifiquement pour les victimes d'AS (Hébert et al., 2006). En effet, l'évitement est un facteur reconnu comme étant central dans la multiplicité des réactions à la suite d'un traumatisme, et ce, tant chez les enfants que chez les adultes victimes (Hébert, 2014). Se sentir coupable par rapport à la situation d'AS incite donc les enfants victimes à utiliser des stratégies pour éviter les pensées et les sentiments liés à l'AS, ce qui augmente les risques de rapporter des symptômes anxieux et une faible estime de soi.

Parmi les caractéristiques de l'AS étudiées, seule la sévérité a été retenue comme prédicteur du sentiment de culpabilité. Le fait d'avoir subi des gestes de pénétration ou de tentative de pénétration était associé à un plus grand sentiment de culpabilité révélé par les enfants victimes. Des relations similaires avaient été observées chez les personnes ayant vécu une AS durant l'enfance (Cantón-Cortés et al., 2011; Feiring et Cleland, 2007; Quas et al., 2003). Toutefois, cette contribution demeure faible. Ceci pourrait être dû à la distribution anormale des données (61,6\% des participants de cette étude ont été victimes d'AS très sévères) qui diminue la variance pouvant être expliquée. Parmi les autres caractéristiques de l'AS étudiées dans la présente étude, la fréquence et l'identité de l'agresseur ne corrélaient pas significativement 
avec le sentiment de culpabilité. Pourtant, des études avaient déjà soulevé qu'une situation d'AS qui perdure dans le temps contribuait à davantage de sentiment de culpabilité chez des victimes d'AS durant l'enfance (Cantón-Cortés et al., 2011; Quas et al., 2003). Il importe de mentionner que les données quant aux caractéristiques des AS vécues, et plus particulièrement la fréquence et les types de gestes subis, peuvent être plus difficiles à répertorier dans le cas d'enfants comparativement aux adultes. En effet, il arrive parfois que les informations quant à la nature exacte ou l'ampleur des différentes situations vécues ne soient dévoilées qu'en cours de thérapie lorsque le lien de confiance se développe. Les données pourraient donc représenter seulement une portion des actes vécus. Cette différence pourrait être également expliquée par l'utilisation d'une échelle catégorielle (épisode unique, plusieurs événements ou fréquence répétitive) plutôt que continue (p. ex: durée en nombre de mois). Par ailleurs, les recherches précédemment recensées soulevaient des contradictions quant au lien entre l'identité de l'agresseur et le sentiment de culpabilité vécu par l'enfant: certaines indiquaient qu'il n'y avait pas de lien significatif entre le sentiment de culpabilité et l'identité de l'agresseur (Cantón-Cortés et al., 2011) tandis que d'autres indiquaient qu'une relation de proximité avec l'agresseur prédisait un plus grand sentiment de culpabilité (Quas et al., 2003). Ces contradictions pourraient s'expliquer par la proximité affective que peuvent avoir l'enfant et l'agresseur, car cette proximité peut complexifier le processus d'attribution du blâme (Feiring et Cleland, 2007). Dans la présente étude, trois participants sur quatre ont été agressés sexuellement par un membre de leur famille. Cette faible variabilité pourrait également expliquer l'absence de lien entre l'identité de l'agresseur et le sentiment de culpabilité.

En ce qui a trait aux différences possibles entre les garçons et les filles, les analyses multigroupes ont révélé que les données s'ajustaient de manière équivalente au modèle pour les deux genres. Feiring et Cleland (2007) avaient également observé que les garçons et les filles s'attribuent autant de blâme, et ce, chez les victimes comme chez les non-victimes. Le modèle médiateur proposé dans la présente étude semble donc valide tant pour les garçons que pour les filles. Ceci souligne l'importance d'intervenir avec les filles comme avec les garçons victimes d'AS, même si ces difficultés (anxiété et estime de soi) sont parfois davantage associées au genre féminin. 
Les résultats de cette étude semblent indiquer que le sentiment de culpabilité et les stratégies d'adaptation seraient des cibles d'intervention pertinentes pour les enfants victimes d'AS. D'ailleurs, la thérapie cognitivo-comportementale axée sur le trauma (Cohen, Mannarino et Deblinger, 2006), qui est reconnue comme une intervention exemplaire dans le domaine des enfants victimes d'AS, inclut une composante sur la reconnaissance des pensées et la restructuration cognitive (Simoneau, Daignault et Hébert, 2011). Dans le cadre des sessions de thérapie, les enfants sont amenés à reconnaître que leurs pensées ont un impact sur la façon dont ils se sentent. Durant la thérapie, les enfants qui mentionnent se sentir coupables de l'AS pourront prendre conscience, à l'aide du soutien du thérapeute, qu'ils ne sont aucunement responsables de cette situation, ce qui pourrait contribuer à diminuer leur sentiment de culpabilité et d'autres symptômes associés à l'AS. De plus, l'utilisation de stratégies d'approche, telles qu'aborder la situation vécue directement, parler de ses émotions et les partager avec le parent non agresseur, est appliquée et promue durant les séances. Des interventions sociales préventives devraient également promouvoir l'attribution du blâme à l'agresseur et l'utilisation de stratégies d'approche efficaces.

\section{Limites de la présente étude et recherches futures}

Bien que cette recherche ait permis de mieux comprendre le rôle du sentiment de culpabilité chez les jeunes victimes d'AS, elle comporte quelques limites. Premièrement, le fait que l'étude soit transversale ne permet pas d'établir de lien de causalité ni de vérifier si l'effet médiateur de l'évitement est constant à travers le temps. Deuxièmement, l'instrument utilisé pour mesurer le sentiment de culpabilité chez les enfants contient un nombre limité d'énoncés. L'utilisation d'une échelle ayant une plus grande sensibilité aurait été un atout. Troisièmement, toutes les mesures ont été remplies par l'enfant. Il aurait été pertinent d'inclure des informations provenant d'un parent non agresseur afin de trianguler les données.

Des recherches ultérieures pourraient étudier l'impact du sentiment de culpabilité sur d'autres conséquences propres à l'AS chez les enfants, comme les SSPT, les symptômes de dissociation ou la dépression. Il serait également intéressant d'inclure un deuxième temps de mesure pour vérifier si les relations entre le sentiment de culpabilité, l'évitement et les symptômes associés à l'AS se maintiennent à travers le temps. 
Puisqu'un dévoilement d'AS peut occasionner une situation de crise pour l'ensemble des acteurs dans l'entourage de l'enfant (p. ex., séparation, perte de contact avec certains membres de la famille), il serait possible de penser que les enfants vivraient davantage de culpabilité peu de temps après le dévoilement. Des chercheurs pourraient également créer des outils de mesure sur les attributions de blâme, le sentiment de culpabilité et la honte qui soient adaptés aux enfants victimes. Des instruments de mesure normalisés propres à cette population permettraient de mieux distinguer ces trois construits et leurs liens avec les symptômes vécus par les enfants victimes.

Cette étude contribue à l'avancement des connaissances scientifiques en incluant un vaste échantillon d'enfants victimes d'AS, dont un nombre important de garçons. De plus, l'échantillon comprend de jeunes victimes âgées de 6 à 12 ans, ce qui permet de mieux comprendre les enjeux associés à l'AS durant ce stade développemental. Somme toute, cette recherche sur le sentiment de culpabilité permet de contribuer à la compréhension de la complexité des symptômes liés à l'AS vécue durant l'enfance et offre certaines pistes à considérer pour quiconque souhaite améliorer les interventions visant cette clientèle.

\section{Références}

Cantón-Cortés, D., Cantón, J., Justicia, F. et Cortés, M. R. (2011). Un modelo de los efectos del abuso sexual infantil sobre el estrés post-traumático: el rol mediador de las atribuciones de culpa y afrontamiento de evitación. Psicothema, 23(1), 66-73.

Causey, D. L. et Dubow, E. F. (1992). Development of a self-report coping measure for elementary school children. Journal of Clinical Child Psychology, 21(1), 47-59.

Cohen, J. A., Mannarino, A. P. et Deblinger, E. (2006). Treating trauma and traumatic grief in children and adolescents. New York, NY: Guilford Press.

Daigneault, I., Tourigny, M. et Hébert, M. (2006). Self-attributions of blame in sexually abused adolescents: A mediational model. Journal of Traumatic Stress, 19(1), 153-157.

Feiring, C. et Cleland, C. (2007). Childhood sexual abuse and abuse-specific attributions of blame over 6 years following discovery. Child Abuse $\bullet-N e g l e c t$, 31(11-12), 1169-1186.

Feiring, C. et Taska, L. S. (2005). The persistence of shame following sexual abuse: A longitudinal look at risk and recovery. Child Maltreatment, 10(4), 337-349.

Feiring, C., Taska, L. S. et Chen, K. (2002). Trying to understand why horrible things happen: Attribution, shame, and symptom development following sexual abuse. Child Maltreatment, 7(1), 25-39. 
Finkelhor, D. et Browne, A. (1985). The traumatic impact of child sexual abuse: A Conceptualization. American Journal of Orthopsychiatry, 55(4), 530-541.

Gagnon, M. M. et Tourigny, M. (2011). Les comportements sexuels problématiques chez les enfants âgés de 12 ans et moins: Évaluation et traitement. Dans M. Hébert, M. Cyr et M. Tourigny (dir.), L'agression sexuelle envers les enfants (Tome 1, p. 333-362). Québec: Les Presses de l'Université du Québec.

Harter, S. (1985). The Self-Perception Profile for Children: Revision of the Perceived Competence Scale for Children (Manuel). Denver, CO: University of Denver.

Hébert, L. (2014). Modelage parental des stratégies d'adaptation chez les enfants victimes d'agression sexuelle. (Mémoire de maîtrise, Université du Québec à Montréal). Répéré à http://www.archipel.uqam.ca/6147/

Hébert, M. (2011). Les profils et l'évaluation des enfants victimes d'agression sexuelle. Dans M. Hébert, M. Cyr et M. Tourigny (dir.), L'agression sexuelle envers les enfants (Tome 1, p. 149-204). Québec: Les Presses de 1'Université du Québec.

Hébert, M., Langevin, R., et Daigneault, I. (2016). The association between peer victimization, PTSD, and dissociation in child victims of sexual abuse. Journal of Affective Disorders, 193, 227-232.

Hébert, M. et Parent, N. (1995). Traduction française du Self-Perception Profile for Children (SPPC; Harter, 1985). Document inédit. Sainte-Foy, Québec: Université Laval.

Hébert, M., Parent, N. et Daignault, I. V. (2007). The French-Canadian version of the Self-Report Coping Scale: Estimates of the reliability, validity, and development of a short form. Measurement and Evaluation in Counseling and Development, 40, 2-15.

Hébert, M. Tremblay, C., Parent, N., Daignault, I. V. et Piché, C. (2006). Correlates of behavioral outcomes in sexually abused children. Journal of Family Violence, 21(5), 287-299.

Heflin, A. H. et Deblinger, E. (2007). Child sexual abuse. Dans F. M. Dattilio et A. Freeman (dir.), Cognitive-behavioral strategies in crisis intervention ( $3^{\mathrm{e}}$ éd., p. 247-276). New York, NY: Guilford Press.

$\mathrm{Hu}, \mathrm{L} .-\mathrm{T}$. et Bentler, P. M. (1999). Cutoff criteria for fit indexes in covariance structure analysis: Conventional criteria versus new alternatives. Structural Equation Modeling, 6(1), 1-55.

Jöreskog, K. G. et Sörbom, D. (1993). LISREL 8: Structural equation modeling with the SIMPLIS command language. Hillsdale, NJ: Lawrence Erlbaum Associates, Inc.

Lazarus, R. S. (1993). Coping theory and research: Past, present, and future. Psychosomatic Medecine, 55, 234-247.

Lewis, H. B. (1971). Shame and guilt in neurosis. Psychoanalytic Review, 58(3), 419-438.

MacKinnon, D. P. et Fairchild, A. J. (2009). Current directions in mediation analysis. Current Directions in Psychological Science, 18(1), 16-20.

Maikovich-Fong, A. K. et Jaffee, S. R. (2010). Sex differences in childhood sexual abuse characteristics and victims' emotional and behavioral problems: findings from a national sample of youth. Child Abuse or Neglect, 34(6), 429-437. 
Major, B., Kaiser, C. R. et McCoy, S. K. (2003). It's not my fault: When and why attributions to prejudice protect self-esteem. Personality and Social Psychology Bulletin, 29(6), 772-781.

Maniglio, R. (2009). The impact of child sexual abuse on health : a systematic review of reviews. Clinical Psychology Review, 29(7), 647-657.

Martin, R. C. et Dahlen, E. R. (2005). Cognitive emotion regulation in the prediction of depression, anxiety, stress, and anger. Personality and Individual Differences, 39(7), 1249-1260.

Muthén, L. K. et Muthén, B. O. (1998-2015). Mplus user's guide. Los Angeles, $\mathrm{CA}$ : Muthén \& Muthén.

Parent, N. et Hébert, M. (2006). Questionnaire sur la victimisation de l'enfant. Adaptation française du "History of Victimization Form» par Wolfe, Gentile et Boudreau (1987). Montréal, Québec: Département de sexologie, Université du Québec à Montréal.

Quas, J. A., Goodman, G. S. et Jones, D. P. H. (2003). Predictors of attributions of self-blame and internalizing behavior problems in sexually abused children. Journal of Child Psychology and Psychiatry, 44(5), 723-736.

Reynolds, C. R. et Richmond, B. O. (1978). What I Think and Feel : A revised measure of children's manifest anxiety. Journal of Abnormal Child Psychology, 6(2), 271-280.

Simoneau, A. C., Daignault, I. V. et Hébert, M. (2011). La thérapie cognitivocomportementale axée sur le trauma. Dans M. Hébert, M. Cyr et M. Tourigny (dir.), L'agression sexuelle envers les enfants (Tome 1, p. 363-398). Québec: Les Presses de l'Université du Québec.

Stoltenborgh, M., van Ijzendoorn, M. H., Euser, E. M. et Bakermans-Kranenburg, M. J. (2011). A global perspective on child sexual abuse: meta-analysis of prevalence around the world. Child Maltreatment, 16(2), 79-101.

Tangney, J. P., Stuewig, J. et Mashek, D. J. (2007). Moral emotions and moral behavior. Annual Review of Psychology, 58, 345-372. doi: 10.1146/annurev. psych.56.091103.070145

Tourigny, M. et Baril, K. (2011). Les agressions sexuelles durant l'enfance: ampleur et facteurs de risque. Dans M. Hébert, M. Cyr et M. Tourigny (dir.), L'agression sexuelle envers les enfants (Tome 1, p. 7-50). Québec: Les Presses de l'Université du Québec.

Turgeon, L. et Chartrand, É. (2003). Reliability and validity of the Revised Children's Manifest Anxiety Scale in a French-Canadian sample. Psychological Assessment, 15(3), 378-383.

Ullman, S. E. et Filipas, H. H. (2005). Gender differences in social reactions to abuse disclosures, post-abuse coping, and PTSD of child sexual abuse survivors. Child Abuse or Neglect, 29(7), 767-782.

Ullman, S. E., Peter-Hagene, L. C. et Relyea, M. (2014). Coping, emotion regulation, and self-blame as mediators of sexual abuse and psychological symptoms in adult sexual assault. Journal of Child Sexual Abuse, 23(1), 74-93.

Ullman, S. E., Townsend, S. M., Filipas, H. H. et Starzynski, L. L. (2007). Structural models of the relations of assault severity, social support, avoi- 
dance coping, self-blame, and PTSD among sexual assault survivors. Psychology of Women Quarterly, 31, 23-37.

Villeneuve Cyr, M. et Hébert, M. (2011). Analyse comparative des caractéristiques de l'agression sexuelle et des conséquences associées en fonction du sexe. Service social, 57(1), 15-30.

Wolfe, V. V. (2002). The Children's Impact of Traumatic Events Scale II (CITES-II). [Instrument d'évaluation non publié]. London, Ontario: London Health Sciences Center.

Wolfe, V. V. (2007). Child Sexual Abuse. Dans E. J. Mash et R. A. Barkley (dir.), Assessment of Childhood Disorders (p. 685-748). New York, NY: The Guilford Press.

Yuan, K. H. et Bentler, P. M. (2000). Three likelihood-based methods for mean and covariance structure analysis with nonnormal missing data. Sociological methodology, 30(1), 165-200.

\title{
Sense of guilt in sexually abused children. The mediating role of avoidance coping on anxiety and self-esteem
}

\begin{abstract}
Prior studies have suggested self-blame and sense of guilt as important elements associated with the effects of childhood sexual abuse on adult survivors (Cantón-Cortés, Cantón, Justicia \& Cortés, 2011). However, few studies have explored the potential impact of the sense of guilt on outcomes in child victims. This study examines the mediating role of avoidance coping on the relationship between sense of guilt and outcomes (anxiety and self-esteem) in sexually abused children. The sample consisted of 447 sexually abused children (319 girls and 128 boys) aged 6 to 12. Path analysis indicated that children with higher feelings of guilt about the abuse showed more anxiety and lower levels of self-esteem. Indirect effects also indicated that sense of guilt predicted avoidance coping, which in turn contributed to higher anxiety and lower self-esteem. This model, which fits the data well for both girls and boys, explains $24.4 \%$ of the variance in anxiety and $11.2 \%$ of the variance in self-esteem. Results suggest that sense of guilt is an important target for clinical intervention with sexually abused children.
\end{abstract}

KEYWORDS - Child sexual abuse, sense of guilt, avoidance coping, anxiety, self-esteem.

\section{Culpabilidad en los niños víctimas de agresión sexual. El rol mediador de las estrategias de evitación sobre la ansiedad y la autoestima}

RESUMEN - Estudios previos sugieren que el sentimiento de culpabilidad es un factor asociado a las repercusiones de la agresión sexual (AS) en los adultos sobrevivientes (Cantón-Cortés, Cantón, Justicia et Cortés, 2011). Sin embargo, muy pocos estudios han explorado el rol potencial del sentimiento de culpabilidad en los sintomas de los niños víctimas. El objetivo de la presente investigación es estudiar el rol mediador de la evitación en la relación entre el sentimiento de culpabilidad y los sintomas asocia- 
dos a la AS (ansiedad y autoestima). La muestra es de 447 niños víctimas de AS (319 niñas y 128 varones) que tienen entre 6 y 12 años. Los resultados de los análisis indican que los niños que revelan más culpabilidad con respecto a la situación de AS, presentan un nivel más elevado de ansiedad y una autoestima a la baja. Un efecto indirecto fue igualmente observado, e indica que el sentimiento de culpabilidad está ligado a la utilización de estrategias de evitación, que exacerban, a su vez, los sintomas de ansiedad y contribuyen a una más baja autoestima. El modelo, que se ajusta a los datos de manera equivalente para las niñas y los varones, permite de explicar $24,4 \%$ de la varianza de los síntomas de ansiedad y $11,2 \%$ de la varianza de la autoestima. Estos resultados sugieren que el sentimiento de culpabilidad podría constituir un objetivo de intervención adecuada para los niños víctimas de AS.

PAlabras CLAVE - Niños víctimas de agresión sexual, sentimiento de culpabilidad, estrategias de evitación, ansiedad, autoestima. 